\title{
Anomalous photocurrent observed in an Fe-ZnS:Fe Schottky diode
}

\author{
B. K. Li, C. Wang, I. K. Sou, W. K. Ge, and J. N. Wang ${ }^{\text {a) }}$ \\ Department of Physics, the Hong Kong University of Science and Technology, Clear Water Bay, Kowloon, \\ Hong Kong, People's Republic of China
}

(Received 14 September 2007; accepted 3 October 2007; published online 22 October 2007)

\begin{abstract}
Anomalous photocurrent was observed in an epitaxial $\mathrm{Fe} / \mathrm{Zn}_{0 .}{ }_{96} \mathrm{Fe}_{0.04} \mathrm{~S}$ Schottky diode grown by molecular beam epitaxy. The temperature dependent decay behavior of the anomalous photocurrent has been studied. A model based on the photoionization of the acceptorlike interface states is proposed to explain this anomalous phenomenon. By fitting the decay curves of the anomalous photocurrent at different temperatures, ionization energy of the interface states is obtained from an Arrhenius plot of the decay time constants. We believe that these interface states are associated with some complex $(\mathrm{Fe}, \mathrm{S})$ defects formed at the $\mathrm{Fe} / \mathrm{ZnFeS}$ interface. (C) 2007 American Institute of Physics. [DOI: 10.1063/1.2801707]
\end{abstract}

Metal/semiconductor Schottky junctions play an important role in electronic and optoelectronic device applications. $^{1,2}$ In cases where magnetic metals are employed, the magnetic Schottky junctions have a great potential to serve as spin injectors and spin detectors in future spintronics applications. ${ }^{3}$ Recently, epitaxially grown metal/ semiconductor Schottky junction has attracted increasing attentions because it has a rather low level of interface impurity contaminations and this cleaner interface allows a better understanding of the basic and intrinsic properties of the junction. ${ }^{4-6}$ For a Schottky diode formed between a metal and $n$-type semiconductor the short-circuit photocurrent is normally directed from the semiconductor to the metal across the interface. If the photon energy $h v$ is larger than the band gap energy $E_{g}$ of the semiconductor, the excited electrons and holes in the depletion region flow in opposite directions due to the build-in electric field. If the energy of the incident photon lies within the range of $\phi_{B}<h v<E_{g}$, where $\phi_{B}$ is the Schottky barrier height, electrons in the metal have a probability being excited across the interface into the semiconductor depletion region to form a photocurrent, although it is much smaller than that due to the interband excitation. We define the above two types of photocurrent as positive or normal photocurrent. However, anomalous photocurrent with an opposite direction was observed in a $\mathrm{GaN} / \mathrm{AlGaN}$ ultraviolet detector ${ }^{7}$ and $\mathrm{AlO}_{x}$-based magnetic tunnel junctions. ${ }^{8}$ The anomalous contribution to the photocurrent in both cases was explained as the formation of double junctions inside the single device and the result of the competition between the normal and anomalous photoresponse of the two junctions. In this work, the anomalous photocurrent originating from a different mechanism is reported in a molecular beam epitaxy (MBE)-grown $\mathrm{Fe} / \mathrm{Zn}_{0.96} \mathrm{Fe}_{0.04} \mathrm{~S}$ Schottky diode. The anomalous photocurrent is produced by photoionization of interface states. Temperature dependent decay behavior of the anomalous photocurrent is studied and the ionization energy of the interface states is obtained.

The $(5 \mathrm{~nm}) \mathrm{Fe} /(100 \mathrm{~nm}) \mathrm{Zn}_{0.96} \mathrm{Fe}_{0.04} \mathrm{~S}$ Schottky structure was grown using a VG V80H MBE system in a single growth run with $\mathrm{ZnS}$ and $\mathrm{Fe}$ sources loaded in two separated

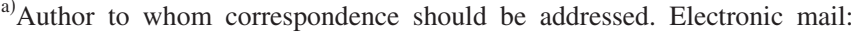
phjwang@ust.hk effusion cells. A $400 \mathrm{~nm} \mathrm{ZnS} \mathrm{buffer} \mathrm{layer} \mathrm{was} \mathrm{first} \mathrm{grown} \mathrm{on}$ the $n^{+}$-GaAs (100) substrate. After the growth of the Fe layer a $3 \mathrm{~nm} \mathrm{Au}$ film was then deposited as a capping layer to prevent oxidization of the Fe layer. Reference samples, such as $\mathrm{Au} / \mathrm{Zn}_{0.96} \mathrm{Fe}_{0.04} \mathrm{~S}, \mathrm{Fe} / \mathrm{ZnS}$, and $\mathrm{Fe} / \mathrm{ZnMgS}$, were also grown under the same conditions. The short-circuit dc photocurrent spectra were measured using an HP 4155A semiconductor parameters analyzer while a $150 \mathrm{~W}$ xenon arc lamp and an SBP300 monochromator were used as the light source. Current-voltage curves and the time decay processes of the photocurrents were also measured using the HP4155A semiconductor parameters analyzer. The integration time was set to be $40 \mathrm{~ms}$ in order to reduce the noise during the photocurrent decay measurements.

The current-voltage curves at 300 and $13 \mathrm{~K}$ of the $\mathrm{Fe} / \mathrm{Zn}_{0.96} \mathrm{Fe}_{0.04} \mathrm{~S}$ structure show the typical characteristics of a Schottky diode formed between metal and $n$-type semiconductor, as shown in Fig. 1(a). Figure 1(c) shows the shortcircuit dc photocurrent spectra of the $\mathrm{Fe} / \mathrm{Zn}_{0.96} \mathrm{Fe}_{0.04} \mathrm{~S}$ Schottky diode at room temperature with the excitation photon energy ranging from 300 to $500 \mathrm{~nm}$. As it can be seen,
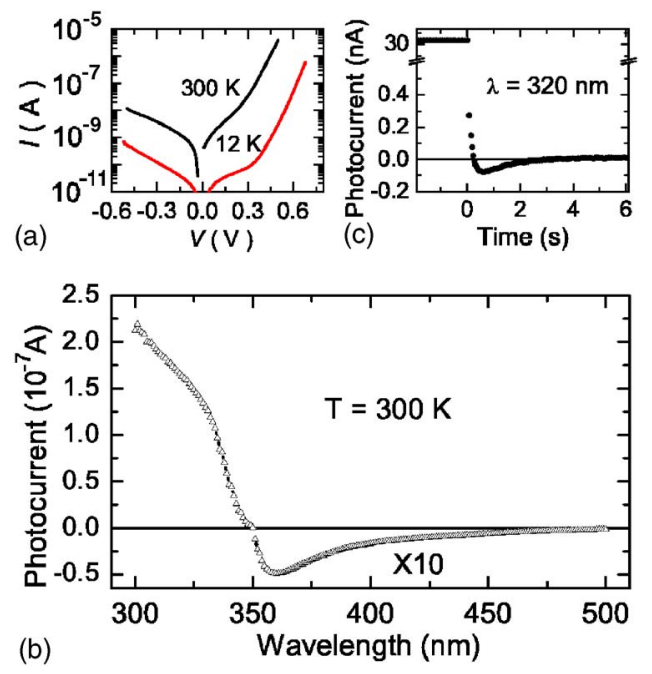

FIG. 1. (Color online) (a) Current-voltage curves of the Fe/ZnFeS Schottky diode measured in dark at 12 and $300 \mathrm{~K}$, respectively. (b) dc photocurrent spectrum of the $\mathrm{Fe} / \mathrm{ZnFeS}$ Schottky diode measured at $300 \mathrm{~K}$. The negative part was enlarged ten times for clarity. (c) Decay curve of the photocurrent generated by a $320 \mathrm{~nm}$ excitation at $300 \mathrm{~K}$. 
the Schottky diode produces a normal dc photocurrent response when incident photon energy is larger than the band gap energy $E_{g}$ of $\mathrm{Zn}_{0.96} \mathrm{Fe}_{0.04} \mathrm{~S}$, which can be estimated by the abrupt fall at around $345 \mathrm{~nm}$ of the dc photocurrent response curves. However, when the incident photon energy is smaller than $E_{g}$, the dc photocurrent shows a remarkable anomaly by changing its direction, i.e., the photocurrent is directed from the metal to the semiconductor. Figure 1(b) shows the transient behavior of the photocurrent generated by a $320 \mathrm{~nm}$ excitation at room temperature. We can see that the anomalous photocurrent concurs with the normal photocurrent but with a much longer decay time constant. For a typical Schottky diode, the decay of the normal photocurrent is via two processes: interband decay (direct recombination of photon-excited electron-hole pairs) with a time constant usually less than a microsecond and defects assisted decay with a time constant much longer, of milliseconds or even longer. ${ }^{9}$ The interband decay is beyond our measurement limit and is not included in the decay curves shown in Fig. 1(b). The measured decay curve results from the competition between the normal photocurrent decay through defects and the anomalous photocurrent decay. So the dc photocurrent spectrum in Fig. 1(c) is the sum of normal and anomalous photocurrents. When photon excitation energy is above the band gap energy of $\mathrm{Zn}_{0.96} \mathrm{Fe}_{0.04} \mathrm{~S} E_{g}$ the normal photocurrent dominates, while it is below $E_{g}$ the anomalous photocurrent dominates.

In order to understand the origins of this anomalous photocurrent, we have measured the photocurrent spectra of the reference samples including $\mathrm{Au} / \mathrm{Zn}_{0.96} \mathrm{Fe}_{0.04} \mathrm{~S}, \mathrm{Fe} / \mathrm{ZnS}$, and $\mathrm{Fe} / \mathrm{ZnMgS}$ Schottky diodes and $\mathrm{Au} / \mathrm{ZnMgS}$ (Ref. 10) and $\mathrm{Au} / \mathrm{ZnSSe}$ (Ref. 11) UV detectors. However, no anomalous photocurrent was detected from all these reference samples. Since these reference samples were grown under the same conditions as the $\mathrm{Fe} / \mathrm{Zn}_{0.96} \mathrm{Fe}_{0.04} \mathrm{~S}$ structure, we can conclude that the anomalous effect is a unique feature related to the interface properties of the $\mathrm{Fe} / \mathrm{Zn}_{0.96} \mathrm{Fe}_{0.04} \mathrm{~S}$ structure. It has been shown that the band gap of $\mathrm{ZnFeS}$ is evidently narrowed with increasing $\mathrm{Fe}$ composition. ${ }^{12}$ This means that if a higher $\mathrm{Fe}$ content layer existed at the interface of the $\mathrm{Fe} / \mathrm{Zn}_{0.96} \mathrm{Fe}_{0.04} \mathrm{~S}$ Schottky diode due to $\mathrm{Fe}$ diffusion from the Fe layer into the $\mathrm{ZnFeS}$ layer, the conduction band bending could be attenuated or even changes its direction in the depletion layer close to the interface. This highly doped layer can generate the anomalous response when the incident photoenergy is below the band gap of $\mathrm{Zn}_{0.96} \mathrm{Fe}_{0.04} \mathrm{~S}$. However, previous investigations of the thermal stability of $\mathrm{Fe}$ on $\mathrm{ZnS}$ matrix using secondary ion mass spectroscopy indicates that $\mathrm{Fe} / \mathrm{ZnS}$ interface is stable at least up to $350{ }^{\circ} \mathrm{C} .{ }^{13}$ In addition, the reference samples of $\mathrm{Fe} / \mathrm{ZnS}$ and $\mathrm{Fe} / \mathrm{ZnMgS}$ Schottky diodes do not show the anomaly. It is thus unlikely a higher $\mathrm{Fe}$ content layer was formed close to the interface in the $\mathrm{Fe} / \mathrm{Zn}_{0.96} \mathrm{Fe}_{0.04} \mathrm{~S}$ structure due to thermal diffusion. On the other hand, the decay behavior of photocurrent generated by a $320 \mathrm{~nm}$ excitation, as shown in Fig. 1(c), and the transient behavior of this anomalous effect as will be discussed later also do not support this assumption.

As a result, we propose that some acceptorlike interface states that can trap photon-excited electrons are responsible for the observed anomalous photocurrent. As can be seen in the schematic band diagram of the Fe/ZnFeS Schottky junction shown in Fig. 2, the interface states are usually continuously distributed in the semiconductor band gap. ${ }^{2}$ When the Downloaded 28 Aug 2008 to 143.89.106.125. Redistribution subje

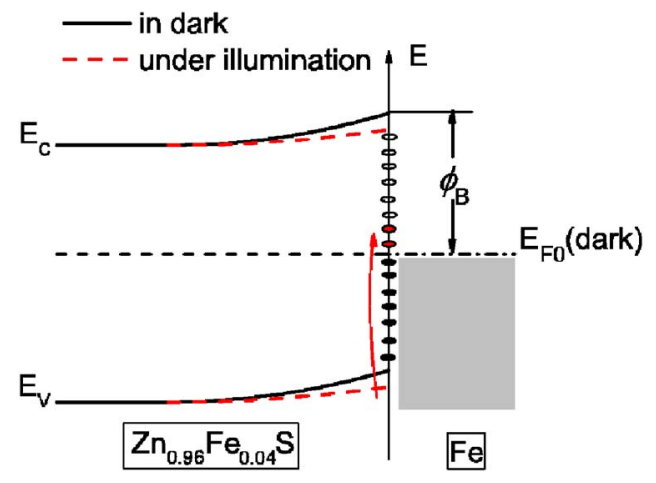

FIG. 2. (Color online) Schematic band diagram of the $\mathrm{Fe} / \mathrm{Zn}_{0.96} \mathrm{Fe}_{0.04} \mathrm{~S}$ Schottky diode in dark (black, solid line) and under illumination (red, dashed line).

diode is in dark and at equilibrium, the Fermi levels of the semiconductor and the metal are aligned. The acceptorlike interface states are filled up to the Fermi level. The interface and the depletion region are electrically neutral. However, when the diode is illuminated by photons with energy $h v>E_{g}-\phi_{B}$, electrons in the valence band at the interface region can be excited to and trapped by the acceptorlike empty interface states above the equilibrium Fermi level. When these acceptorlike interface states trap electrons they become negatively charged. In comparison with the equilibrium situation, the interface under illumination is more negatively charged. In other words, the light illumination results in a shift of the Fermi level at the interface region toward the conduction band of $\mathrm{ZnFeS}$ and reduce the band bending in the depletion region, as indicated in Fig. 2. This is equivalent to applying a small forward bias across the diode. Therefore, the photocurrent produced by the photoexcitation of acceptorlike interface states is in opposite direction to the normal photocurrent.

When light is turned off, the interface returns to its equilibrium by releasing the electrons trapped at the interface states. Generally speaking, the process of releasing the electrons trapped at the interface states is expected to be slow. Figure 3(a) shows the measured decays of the anomalous photocurrent generated by photons of $362 \mathrm{~nm}$. It indicates that the trapping time constant is in order of seconds. Such a long trapping time rules out the previously discussed possibility that a high $\mathrm{Fe}$ content layer formed close to the interface is responsible for the anomalous photocurrent. It also suggests that these acceptorlike interface states are rather complicated and we believe that they are associated with $(\mathrm{Fe}, \mathrm{S})$ complex formed at the $\mathrm{Fe} / \mathrm{ZnFeS}$ interface. The exact nature of $(\mathrm{Fe}, \mathrm{S})$ complex is unknown. The fact that $\mathrm{Fe} / \mathrm{ZnS}$ Schottky diode does not exhibit anomalous photocurrent effect implies that the existence of $\mathrm{Fe}$ in the semiconductor $\mathrm{ZnS}$ is important to the formation of the $(\mathrm{Fe}, \mathrm{S})$ complex.

The decay process of the anomalous photocurrent can be modeled quantitatively based on the occupation of interface states. We assume that the effective forward bias originating from the trapping of photoexcited electrons by the interface states is very small and is proportional to the number of electrons trapped at the interface,

$$
V_{\text {eff }}=B_{0}^{*} Q,
$$

where $B_{0}^{*}$ is a proportional constant and $Q$ is the number of photoexcited electrons trapped at the interface. While the to AIP license or copyright; see http://apl.aip.org/apl/copyright.jsp 

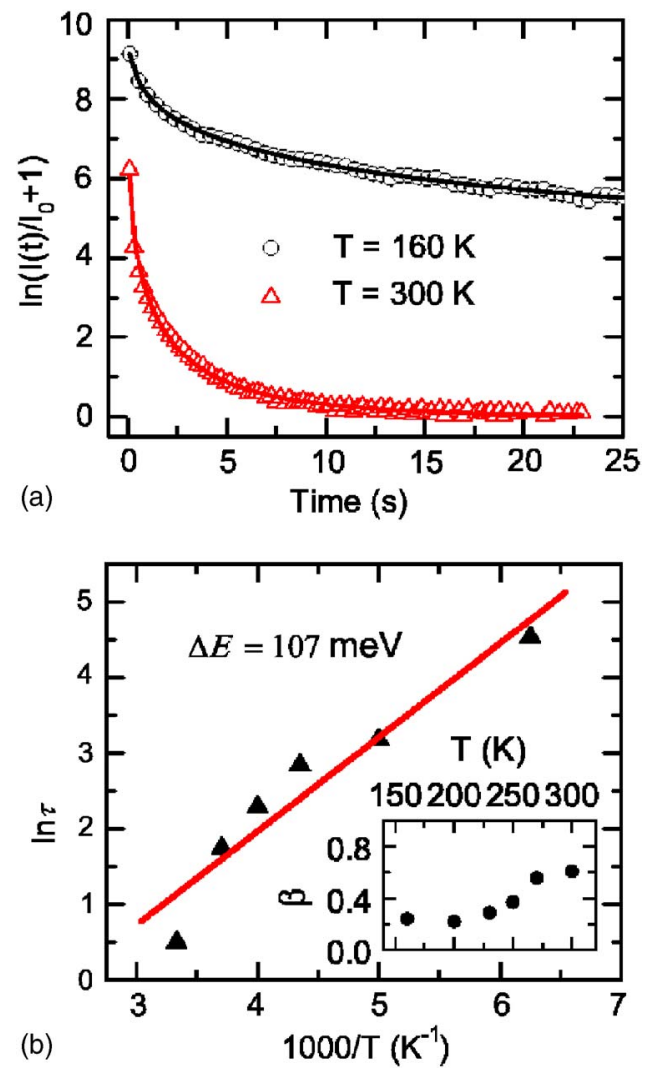

FIG. 3. (Color online) (a) Experimental (symbols) and fitted (solid lines) decay curves of the anomalous photocurrent excited by $362 \mathrm{~nm}$ light at different temperatures as indicated. (b) Arrhenius plot of the decay time constants and solid line is a linear fit. The inset shows the decay exponent at different temperatures.

decay of $Q$ follows a stretched-exponential relaxation law, ${ }^{14}$

$$
Q(t)=Q_{0} \exp \left[-(t / \tau)^{\beta}\right] \quad(0<\beta<1),
$$

where $\tau$ is the stretched-exponential decay time, $\beta$ is the decay exponent, and $Q_{0}$ is the number of electrons trapped by the interface states at an excitation equilibrium. For a Schottky diode, the current under a bias follows ${ }^{1}$

$$
\begin{aligned}
& I=I_{0}[\exp (q V / n k T)-1], \\
& I_{0}=A^{*} T^{2} \exp \left(-q \phi_{B} / k T\right),
\end{aligned}
$$

where $A^{*}$ is the effective Richardson constant, $n$ is the ideality factor, $q$ the electron charge, $k$ is the Boltzmann constant, and $\phi_{B}$ is the Schottky barrier height. So by substituting Eqs. (1) and (2) into Eq. (3), the decay of the anomalous photocurrent at different temperatures can be readily calculated. The experimental decay curves can be fitted by

$$
\ln \left[\frac{I(t)}{I_{0}}+1\right]=B_{0} \exp \left[-(t / \tau)^{\beta}\right]
$$

where $B_{0}=B_{0}^{*} q / n k T$ and $I_{0}$ can be obtained from the $I-V$ curves. The solid lines in Fig. 3(a) are the fitted curves using Eq. (4). It is evident that our model produces an excellent fit to the transient behavior of the anomalous photocurrent at different temperatures. This implies that the anomalous photocurrent indeed originates from the occupation of the interface states by the excited electrons. Figure 3(b) is an Arrhenius plot of the obtained decay time constants and the inset shows the decay exponents at different temperatures. The ionization energy of the trapped electrons at the interface states is estimated to be $107 \mathrm{meV}$ from the solid line fit in Fig. 3(b).

In conclusion, through a detailed study on the transient behavior of the photoresponse, the anomalous photocurrent observed in an $\mathrm{Fe} / \mathrm{Zn}_{0.96} \mathrm{Fe}_{0.04} \mathrm{~S}$ Schottky diode is believed to originate from the photoionization of the acceptorlike interface states. The ionization energy of the trapped electrons at the interface states is estimated from the temperature dependent decay time constants of the anomalous photocurrent to be $107 \mathrm{meV}$.

This work was supported by the Reseach Grant Council of Hong Kong SAR government via Grant Nos. N-HKUST611/05 and 603704.

${ }^{1}$ S. M. Sze and K. Ng, Physics of Semiconductor Devices, 3rd ed. (Wiley, Hoboken, NJ, 2007), p. 134.

${ }^{2}$ B. L. Sharma, Metal-Semiconductor Schottky Barrier Junctions and Their Applications (Plenum, New York, 1984), p. 1.

${ }^{3}$ I. Zutić, J. Fabian, and S. Das Sarma, Rev. Mod. Phys. 76, 323 (2004).

${ }^{4}$ M. Eddrief, M. Marangolo, S. Corlevi, G.-M. Guichar, V. H. Etgens, R.

Mattana, D. H. Mosca, and F. Sirotti, Appl. Phys. Lett. 81, 4553 (2002).

${ }^{5}$ C. H. Lei, Thin Solid Films 515, 3584 (2007).

${ }^{6}$ R. T. Tung, J. Vac. Sci. Technol. B 11, 1546 (1993).

${ }^{7}$ A. Teke, S. Dogan, L. He, D. Huang, F. Yun, M. Mikkelson, H. Morkoç, S. K. Zhang, W. B. Wang, and R. R. Alfano, J. Electron. Mater. 32, 307 (2003).

${ }^{8}$ P. H. P. Koller, F. W. M. Vanhelmont, H. Boeve, R. Coehoorn, and W. J. M. de Jonge, J. Appl. Phys. 93, 8549 (2003).

${ }^{9}$ E. Monroy, F. Vigué, F. Calle, J. I. Izpura, E. Muñoz, and J.-P. Faurie, Appl. Phys. Lett. 77, 2761 (2000).

${ }^{10}$ I. K. Sou, M. C. W. Wu, T. Sun, K. S. Wong, and G. K. L. Wong, Appl. Phys. Lett. 78, 1811 (2001).

${ }^{11}$ I. K. Sou, Z. H. Ma, and G. K. L. Wong, Appl. Phys. Lett. 75, 3707 (1999).

${ }^{12}$ Q. J. Feng, D. Z. Shen, J. Y. Zhang, Y. M. Lu, Y. C. Liu, and X. W. Fan, Mater. Chem. Phys. 96, 158 (2006).

${ }^{13}$ I. K. Sou, C. Wang, S. K. Chan, and G. K. L. Wong, J. Cryst. Growth 278, 282 (2005).

${ }^{14}$ R. G. Palmer, D. L. Stein, E. Abrahams, and P. W. Anderson, Phys. Rev. Lett. 53, 958 (1984). 